\title{
A KÜNNETH FORMULA FOR COPRODUCTS OF SIMPLICIAL GROUPS
}

\author{
ELYAHU KATZ
}

\begin{abstract}
Berstein [1] (and later Clark and Smith [9]) proved that over a field the homology of the coproduct of two simplicial groups is isomorphic to the coproduct of the augmented algebras obtained by taking the homology of these groups. It turns out that this result does not hold in general over the integers. However, we obtain a Künneth like formula in which the homology of the coproduct of two groups is expressed in terms of the homology of the groups.
\end{abstract}

1. Introduction. The paper consists of two results whose combination establishes the main result.

Let $R\left(G_{i}\right)$ be the augmented differential graded algebras over a commutative ring $R$, associated with the simplicial groups $G_{i}, i=1,2$. We prove that $R\left(G_{1} * G_{2}\right)$ is chain homotopy equivalent to $R\left(G_{1}\right) \amalg R\left(G_{2}\right)$, where * stands for the coproduct of the groups and $\amalg$ stands for the coproduct of differential augmented algebras (see $\$ 2$ ).

The second result is a Künneth like formula for coproducts. More precisely $H\left(Z\left(G_{1}\right) \amalg Z\left(G_{2}\right)\right)$ is expressed in terms of $H\left(Z\left(G_{1}\right)\right)$ and $H\left(Z\left(G_{2}\right)\right)$ and a torsion factor denoted by mult $\left(G_{1}, G_{2}\right)$ (see $\left.\$ 4\right)$, where $Z$ is the integers.

The main result provides us with a formula in which $H\left(Z\left(G_{1} * G_{2}\right)\right)$ is isomorphic to the direct sum of $H\left(Z\left(G_{1}\right)\right) \amalg H\left(Z\left(G_{2}\right)\right)$ and mult $\left(G_{1}, G_{2}\right)$. This can be interpreted as follows: Let $X_{1}$ and $X_{2}$ be well-pointed, connected, topological spaces. Then, $H\left(\Omega\left(X_{1} \vee X_{2}\right)\right)$ can be expressed in terms of $H\left(\Omega\left(X_{1}\right)\right)$ and $H\left(\Omega\left(X_{2}\right)\right)$. Our application of the latter is restricted to some trivial cases in which mult $=0$. In particular, we obtain $H\left(\Omega\left(S^{l} \vee S^{k}\right)\right)$ $\cong H\left(\Omega\left(S^{l}\right)\right) \amalg H\left(\Omega\left(S^{k}\right)\right), k, l>1$.

2. An Eilenberg-Zilber theorem for coproducts. In [1] coproducts in the category of connected graded algebras were defined. This definition can be extended to differential augmented graded algebras. For the convenience of the reader we state the definition for the more general case, explicitly. However, we start with some other definitions.

Definition 1. Let $G_{1}$ and $G_{2}$ be objects of $\mathcal{G}$ the category of simplicial groups. Their coproduct $G_{1} * G_{2}$ is defined as follows: $\left(G_{1} * G_{2}\right)_{n}=\left(G_{1}\right)_{n}$

Received by the editors February 13, 1976.

AMS (MOS) subject classifications (1970). Primary 57F99; Secondary 55J25, 55J10.

Key words and phrases. Coproduct of simplicial groups, coproduct of differential graded algebras, acyclic models. 
* $\left(G_{2}\right)_{n}$, where $(G)_{n}$ is the group of all elements of $G$ of degree $n$, and * of the right-hand side of the equation stands for the free product of groups. The degeneracy and boundary operators of $G_{1} * G_{2}$ are induced by those of $G_{1}$ and $G_{2}$.

Definition 2. The associated algebra $R(G)$ of the simplicial group $G$ is defined as follows: $(R(G))_{n}$ is the free $R$ module generated by $(G)_{n}$, and the boundary operator on the generators is defined by $\partial g=\sum_{i=0}^{n}(-1)^{i} \partial_{i} g, g$ $\in(G)_{n}$.

Definition 3. Let $A_{1}$ and $A_{2}$ be objects of $\mathscr{Q}$ the category of augmented differential graded algebras over the ring $R$. Their coproduct is defined by $A_{1} \amalg A_{2}=R \oplus \sum_{I} \bar{A}_{I}$, where $I$ exhausts the collection of all sequences alternating on 1 and 2 . If $I=\left(j_{1}, j_{2}, \ldots, j_{n}\right)$, then $\bar{A}_{I}=\bar{A}_{j_{1}} \otimes \bar{A}_{j_{2}} \otimes \cdots \otimes \bar{A}_{j_{n}}$ where $\bar{A}_{j_{k}}$ is the augmentation of $A_{j_{k}}$. The algebra structure of $A_{1} \amalg A_{2}$ is induced by the algebra structures of $A_{1}$ and $A_{2}$ and the tensor product.

Definitions 1 and 3 extend trivially to any finite number of objects.

THEOREM 1. Let $G_{1}, G_{2} \in \mathcal{G}$. Then $R\left(G_{1} * G_{2}\right)$ is chain homotopy equivalent to $R\left(G_{1}\right) \amalg R\left(G_{2}\right)$.

In Theorem 1 we can replace $R()$ by $R_{N}()$, the normalized chain complex of $R()$. The proof is similar to the proof of Corollary 29.4 of [6] and will be omitted.

3. Proof of Theorem 1. The proof of the theorem is based on the method of acyclic models (see, for example, [6]). Thus we turn to the definition of a suitable category, models and functors.

Let $\mathcal{H}$ denote the category of finite ordered tuples of simplicial groups $\left(G_{1}, G_{2}, \ldots, G_{m}\right)$. A morphism from $\left(G_{1}, \ldots, G_{m}\right)$ to $\left(H_{1}, \ldots, H_{n}\right)$ is a tuple $\left(h_{1}^{j_{1}}, \ldots, h_{m}^{j_{m}}\right)$ such that $h_{i}^{j_{i}}: G_{i} \rightarrow H_{j_{i}}$ is a homomorphism, $1 \leqslant i \leqslant m, 1 \leqslant j_{i}$ $\leqslant n$. If also $\left(k^{r_{1}}, \ldots, k_{n}^{r_{n}}\right):\left(H_{1}, \ldots, H_{n}\right) \rightarrow\left(K_{1}, \ldots, K_{p}\right)$ is a morphism in $\mathcal{H}$, the superposition $\left(k_{1}, \ldots, k_{n}\right)\left(h_{1}, \ldots, h_{m}\right)=\left(l_{1}, \ldots, l_{m}\right)$ is defined as follows: $l_{t}^{s_{t}}=k_{j_{t}}^{s_{t}} h_{t}^{j_{t}}: G_{t} \rightarrow K_{s_{t}}, 1 \leqslant t \leqslant m$.

We define two functors $\alpha, \beta: \mathcal{H} \rightarrow \mathcal{Q}$. On objects,

$$
\alpha\left(G_{1}, \ldots, G_{m}\right)=R\left(G_{1} * \cdots * G_{m}\right)
$$

and on morphisms if $h_{1} * \cdots * h_{m}: G_{1} * \cdots * G_{m} \rightarrow H_{1} * \cdots * H_{n}$ is the obvious homomorphism, then $\alpha\left(h_{1}, \ldots, h_{m}\right)=R\left(h_{1} * \cdots * h_{m}\right)$ is the chain map induced by the simplicial homomorphism. The other functor is defined on objects by $\beta\left(G_{1}, \ldots, G_{m}\right)=R\left(G_{1}\right) \amalg \cdots \amalg R\left(G_{m}\right)$, and on morphisms by $\beta\left(h_{1}, \ldots, h_{m}\right)=\alpha\left(h_{2}\right) \amalg \cdots \amalg \alpha\left(h_{m}\right)$.

We choose the following models for $\mathcal{H}$. Let $\Delta_{n}$ be the standard complex with one nondegenerate element $\delta_{n}$ of degree $n$. Denote by $M_{n}=F(\Delta n)$ Milnor's free group construction on $\Delta n$ [7]. The models of our category are all tuples $\left(M_{i_{1}}, M_{i_{2}}, \ldots, M_{i_{m}}\right)=M_{i_{1}, i_{2}}, \ldots, i_{m}$.

Proposition 1. $\alpha\left(M_{i_{1}}, \ldots, i_{m}\right)$ is acyclic, i.e. 


$$
H_{n}\left(\alpha\left(M_{i_{1}}, \ldots, i_{m}\right)\right)= \begin{cases}0, & n \neq 0 \\ R, & n=0 .\end{cases}
$$

From the isomorphism $F\left(\Delta_{i_{1}}\right) * \cdots * F\left(\Delta_{i_{m}}\right) \cong F\left(\Delta_{i_{1}} \vee \cdots \vee \Delta_{i_{m}}\right)$ which follows from the definition of the $F$ construction, we have $H\left(\alpha\left(M_{i_{1}}, \ldots, i_{m}\right)\right)$ $=H\left(F\left(\Delta_{i_{1}} \vee \cdots \vee \Delta_{i_{m}}\right)\right)$. Since $\Delta_{i_{k}}$ is contractible for each $k$, so is $\Delta_{i_{1}} \vee \cdots$ $\vee \Delta_{i_{m}}$. Because of the commutativity of the order of applying the geometric realization and loop and suspension constructions, we obtain the contractibility of $F\left(\Delta_{i_{1}} \vee \cdots \vee \Delta_{i_{m}}\right)=G E\left(\Delta_{i_{1}} \vee \cdots \vee \Delta_{i_{m}}\right)$. ( $G$ is the simplicial loop construction and $E$ the suspension construction. For details see [6].)

Proposition 2. $\beta\left(M_{i_{1}}, \ldots, i_{m}\right)$ is acyclic.

We defined $\beta\left(M_{i_{1}}, \ldots, i_{m}\right)=R\left(M_{i_{1}}\right) \amalg \cdots \amalg R\left(M_{i_{m}}\right)=R \oplus \Sigma_{I} \Lambda_{I}$, where $\Lambda_{I}$ stands for the following complex: $I=\left(j_{1}, \ldots, j_{n}\right)$ belongs to the collection of all sequences in the integers $1,2, \ldots, m$, such that adjoint integers differ from each other. $\Lambda_{I}$ stands for $\bar{R}\left(M_{j_{1}}\right) \otimes \cdots \otimes \bar{R}\left(M_{j_{m}}\right)$ where $\bar{R}(M)$ is the augmented complex of $R(M)$. Homology commutes with direct sums. Thus we need to compute $H\left(\Lambda_{I}\right)$. However, because of the associativity of the tensor product and the fact that $H\left(\bar{R}\left(M_{k}\right)\right)=0$ for all $k$, we obtain from the Künneth formula for two complexes, that $H\left(\Lambda_{I}\right)=0$. We conclude that $H\left(\beta\left(M_{i_{1}}, \ldots, i_{m}\right)\right)=R$ $\oplus \Sigma_{I} H\left(\Lambda_{I}\right)=R$.

Proposition 3. $\alpha$ is representable for $l \geqslant 0$.

Let $r a_{1} \cdot a_{2} \cdots a_{n} \in\left(R\left(G_{1} * \cdots * G_{m}\right)\right)_{l}$ where $r \in R$ and $a_{i} \in\left(G_{k_{i}}\right)_{l}$. Consider the maps $x_{i}: \Delta_{l} \rightarrow G_{k_{i}}$ such that $x_{i}\left(\delta_{l}\right)=$ ai. We denote by $h_{i}^{k_{i}}$ the extension of $x_{i}$ to $F\left(\Delta_{l}\right)$. Then, $\alpha\left(h_{1}^{k_{1}}, \ldots, h_{n}^{k_{n}}\right)\left(\delta_{l} \cdot \delta_{l} \cdots \delta_{l}\right)=a_{1} a_{2} \cdots a_{n}$.

Proposition 4. $\beta$ is representable for $l \geqslant 0$.

For a given element $b_{1} \otimes b_{2} \otimes \cdots \otimes b_{n} \in R\left(G_{1}\right) \amalg \cdots \amalg R\left(G_{n}\right)$ with $b_{i} \in\left(G_{k_{i}}\right)_{l_{i}}$, we define the maps $y_{i}: \Delta_{l_{i}} \rightarrow\left(G_{k_{i}}\right)_{l_{i}}$ such that $y_{i}\left(\delta_{l_{i}}\right)=b_{i}$. The extension of $y_{i}$ to $F\left(\Delta_{l_{i}}\right)$ is denoted by $t_{i}^{k_{i}}$. With this notation we obtain at once that $\beta\left(t_{1}, \ldots, t_{n}\right)\left(\delta_{l_{1}} \otimes \cdots \otimes \delta_{l_{n}}\right)=b_{1} \otimes \cdots \otimes b_{n}$.

ProOf OF THEOREM 1. Define the following maps:

$$
\begin{aligned}
& f_{0}: \alpha\left(G_{1}, \ldots, G_{m}\right) \rightarrow \beta\left(G_{1}, \ldots, G_{m}\right), \\
& g_{0}: \beta\left(G_{1}, \ldots, G_{m}\right) \rightarrow \alpha\left(G_{1}, \ldots, G_{m}\right),
\end{aligned}
$$

by

$$
\begin{gathered}
f_{0}\left(r g_{1} g_{2}, \ldots, g_{n}\right)=r g_{1} \otimes g_{2} \otimes \cdots \otimes g_{n}, \\
g_{0}\left(r g_{1} \otimes g_{2} \otimes \cdots \otimes g_{n}\right)=r g_{1} g_{2} \cdots g_{n},
\end{gathered}
$$

where $r \in R$ and $g_{i} \in G_{k_{i}}$. These maps preserve augmentation, and the result now follows from the method of acyclic models.

4. A generalized Künneth formula. The classical Künneth formula for two chain complexes was extended by Mac Lane [5], Bockstein [2] and Hungerford 
[3] for more than two chain complexes. Here, we use the notation and a special case of the main result of the latter.

THEOREM (HUNGERFORD). Let $K^{1}, K^{2}, \ldots, K^{n}$ be chain complexes of free abelian groups. Then

$$
\begin{aligned}
H_{k}\left(K^{1} \otimes \cdots \otimes K^{n}\right)=\sum_{i=1}^{n-1} \sum_{p_{1}+\cdots+p_{n}+i=k} \operatorname{mult}_{i}^{n}\left(H_{p_{1}}\left(K^{1}\right), \ldots, H_{p_{n}}\left(K^{n}\right)\right) \\
\oplus\left(H\left(K^{1}\right) \otimes \cdots \otimes H\left(K^{n}\right)\right)_{k} .
\end{aligned}
$$

In the theorem the following notation is used:

$$
\operatorname{mult}_{i}^{n}\left(A^{1}, \ldots, A^{n}\right)=H_{i}\left(L^{1} \otimes \cdots \otimes L^{n}\right),
$$

where $L^{r}$ is a free resolution of the abelian group $A^{r}$.

At this point we would like to express the homology of $Z\left(G_{1}\right) \amalg Z\left(G_{2}\right)$ in terms of the homology of $Z\left(G_{1}\right)$ and $Z\left(G_{2}\right)$. Again, the fact that homology commutes with direct sums reduces the problem to computing the homology of multiple tensor products. For this reason we introduce the following notation:

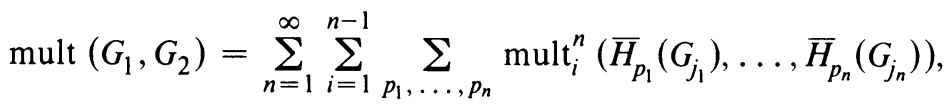

where $p_{1}, \ldots, p_{n}$ are nonnegative integers and $\left(j_{1}, \ldots, j_{n}\right)$ alternate on 1 and 2. (For each $n$ there are exactly two sequences $\left(j_{1}, \ldots, j_{n}\right)$, one starting with 1 and one starting with 2.) It should be noted that the dimension of an element in mult depends only on $i$ and $p_{1}, \ldots, p_{n}$.

\section{THEOREM 2.}

$$
H\left(Z\left(G_{1}\right) \amalg Z\left(G_{2}\right)\right)=Z \oplus H\left(\bar{Z}\left(G_{1}\right)\right) \amalg H\left(\bar{Z}\left(G_{2}\right)\right) \oplus \text { mult }\left(G_{1}, G_{2}\right) .
$$

This follows from a multiple use of Hungerford's theorem.

Theorem 1 with $R=Z$ and Theorem 2 provide us with the main result.

TheOrem 3. $H\left(G_{1} * G_{2}\right)=Z \oplus H\left(\bar{Z}\left(G_{1}\right)\right) \amalg H\left(\bar{Z}\left(G_{2}\right)\right) \oplus$ mult $\left(G_{1}, G_{2}\right)$.

5. Applications. (a) For any two simplicial groups and thus also for any two topological groups $G_{1}$ and $G_{2}$ with torsion-free integral homology, we obtain from Theorem 3: $H\left(G_{1} * G_{2}\right)=H\left(G_{1}\right) \amalg H\left(G_{2}\right)$, because mult $\left(G_{1}, G_{2}\right)$ $=0$.

(b) For well-pointed, connected, topological spaces $X_{1}$ and $X_{2}$, we have the following formula:

$$
\bar{H}\left(\Omega\left(X_{1} \vee X_{2}\right)\right)=H\left(\Omega\left(X_{1}\right)\right) \amalg H\left(\Omega\left(X_{2}\right)\right) \oplus \operatorname{mult}\left(\Omega\left(X_{1}\right), \Omega\left(X_{2}\right)\right) .
$$

We derive the above from Theorem 3 by observing that $\Omega X$ can be replaced by a topological group under quite general conditions [8], and that $G_{1} * G_{2}$ is 
of the homotopy type of $\Omega\left(B_{G_{1}} \vee B_{G_{2}}\right)$ [4]. If the loop spaces of $X_{1}$ and $X_{2}$ have torsion free integral homology, we deduce, as in (a), that

$$
H\left(\Omega\left(X_{1} \vee X_{2}\right)\right)=H\left(\Omega\left(X_{1}\right)\right) \amalg H\left(\Omega\left(X_{2}\right)\right) .
$$

(c) A particular case of (b) is when $X_{1}=S^{l}, X_{2}=S^{k}, l, k>1$. Since for $r>1$,

$$
H_{n}\left(\Omega S^{r}\right)= \begin{cases}Z, & n=0(\bmod r-1) \\ 0 & \text { otherwise }\end{cases}
$$

we get

$$
H\left(\Omega\left(S^{l} \vee S^{k}\right)\right)=H\left(\Omega\left(S^{l}\right)\right) \amalg H\left(\Omega\left(S^{k}\right)\right) .
$$

\section{REFERENCES}

1. I. Berstein, On co-groups in the category of graded algebras, Trans. Amer. Math. Soc. 115 (1965), 257-269. MR 34 \#6757.

2. M. Bockšteīn, A multiple Künneth formula, Izv. Akad. Nauk SSSR Ser. Mat. 27 (1963), 467-482. (Russian) MR 27 \# 1482.

3. T. W. Hungerford, Multiple Künneth formulas for abelian groups, Trans. Amer. Math. Soc. 118 (1965), 257-275. MR 31 \#229.

4. D. M. Kan, A combinatorial definition of homotopy groups, Ann. of Math. (2) 67 (1958), 282-312. MR 22 \# 1897.

5. S. Mac Lane, Triple torsion products and multiple Künneth formulas, Math. Ann. 140 (1960), 51-64. MR 22 \#5661.

6. J. P. May, Simplicial objects in algebraic topology, Van Nostrand, Princeton, N. J., 1967. MR 36 \#5942.

7. J. W. Milnor, Construction of universal bundles. I, Ann. of Math. (2) 63 (1956), 272-284. MR $17,994$.

8. - The construction FK(A.student's guide to algebraic topology by J. Adams), London Math. Soc. Lecture Notes 4 (1972).

9. A. Clark and L. Smith, The rational homotopy of a wedge, Pacific J. Math. 24 (1968), 241-246. MR 36 \# 5945.

Department of Mathematics, University of Haifa, Mount Carmel, Haifa 31999, Israel 